\title{
RANDOM ATTRACTOR FOR A STOCHASTIC HYDRODYNAMICAL EQUATION IN HEISENBERG PARAMAGNET ON AN UNBOUNDED DOMAIN*
}

\author{
Y. F. GUO ${ }^{\dagger}$, B. L. GUO ${ }^{\ddagger}$ D. L. LI ${ }^{\S}$, AND C. X. GUO
}

\begin{abstract}
In this paper, the asymptotic behavior of the stochastic hydrodynamical equation in the Heisenberg paramagnet on the entire two-dimensional space is studied. The asymptotic compactness of the stochastic dynamical system is proved by using the uniform a priori estimates for the far-field values of the solution. The existence of a random attractor is established for the corresponding stochastic dynamical system, and the regularity of the random attractor is obtained, which implies the asymptotic smoothing effect of the equation in a probability sense.
\end{abstract}

Key words. Stochastic partial differential equations, asymptotic compactness, random attractors.

AMS subject classifications. 35Q35, 60H15.

\section{Introduction}

In this paper, we consider the following stochastic hydrodynamical equation emerging from the Heisenberg paramagnet with additive noise defined in the entire space $\mathbb{R}^{2}$ :

$$
d u+(\lambda u-\Delta u-u \times \Delta u) d t=\sum_{i=1}^{m} \phi_{i}(x) d w_{i}(t), \quad x \in \mathbb{R}^{2},
$$

where $\lambda$ is a positive constant, $\phi_{i}(x)(i=1,2, \cdots, m)$ are given smooth functions defined on $\mathbb{R}^{2}$, and $\left\{w_{i}\right\}_{i=1}^{m}$ are independent two-sided real-valued Wiener processes on a probability space which will be specified later. In this equation, $u=\left(u_{1}, u_{2}, u_{3}\right)$ is the unknown denoting the spin density and $\times$ denotes the cross product for vectors in $\mathbb{R}^{3}$. In physics, this equation describes the hydrodynamics of the Heisenberg paramagnet in the long wavelength-low frequency limit when random spatio-temporal forcing is taken into account [12].

In the deterministic case, the authors in [7] studied the equation and obtained the existence and uniqueness of smooth solutions for this equation. This equation is very similar to the Landau-Lifshitz equation [7, 12], which plays an important role in understanding the dynamics of the ferromagnetism in materials, when the temperature is below a critical temperature known as the Curie temperature. One can refer to [10] for a thorough introduction. It is well known that stochastic differential equations play an important role in understanding nonlinear phenomena. Indeed the deterministic model usually neglects the impact of many small perturbations, but

${ }^{*}$ Received: December 28, 2010; accepted (in revised version): March 27, 2011. Communicated by Jack Xin.

${ }^{\dagger}$ Institute of Applied Physics and Computational Mathematics, P.O. Box 8009, Beijing, 100088, China Department of Information and Computation of Science, Guangxi University of Technology, Guangxi, 545006, China (guoyan_feng@yahoo.com.cn; guoyan_feng@163.com).

${ }^{\ddagger}$ Institute of Applied Physics and Computational Mathematics, P.O. Box 8009, Beijing, 100088, China (gbl@iapcm.ac.cn).

$\S$ Department of Information and Computation of Science, Guangxi University of Technology, Guangxi, 545006, China (lidl@21cn.com).

`Department of Mathematics, China University of Mining and Technology Beijing, Beijing, 100083, China (guochunxiao1983@sina.com). 
stochastic equations can conform to physical phenomena better. Recently these problems were intensively investigated by many mathematicians due to the importance of the stochastic equations from the view point of mathematical physics. For example, the stochastic Navier-Stokes equations, KdV, Schrödinger and Burgers' equations are studied $[3,4,5,13]$. In particular, the existence of global smooth solutions was obtained for (1.1) with additive noise on a bounded domain [8].

In order to capture the essential dynamics of stochastic dynamical system, the concept of a random attractor was introduced in $[5,6]$ as the extension to stochastic systems of the theory of attractors for deterministic equations founded in [16, 15]. The random attractor is a compact invariant set depending on chance and moving with time, attracting any orbit starting from $-\infty$. The existence of the random attractor has been studied for stochastic PDEs on bounded domain [5, 6]. For (1.1), the existence of the random attractor has been studied in [9] on bounded subdomains of $\mathbb{R}^{2}$. But the existence of the random attractor has not been studied in the entire space $\mathbb{R}^{2}$ for (1.1).

We know that it is more difficult to prove the existence of random attractors for stochastic PDEs than to prove the existence of attractors for deterministic PDEs on unbounded domains because Sobolev embeddings are no longer compact, and so the compactness of solutions cannot be obtained by the standard method. In the case of stochastic PDEs, in order to overcome this difficulty in unbounded domains we provide the uniform estimates on the far-field values of solutions. This idea was developed in [17] to prove the asymptotic compactness for the deterministic version. By extending the method of using the tail estimates to the case of stochastic dissipative PDEs, some authors have proved the existence of the random attractor on an unbounded domain $[1,18]$. In this paper we use this method to prove the existence of the random attractor in the entire space $\mathbb{R}^{2}$ for (1.1). It is difficult to control the estimates which are produced by the nonlinear term. In order to overcome this difficulty we will use the ergodic property in the process of uniform estimates. The asymptotic compactness of the stochastic dynamical system is proved by using the uniform a priori estimates for the far-field values of solutions via a truncation function.

This paper is organized as follows. In the next section, we recall some fundamental concepts of random attractors for stochastic dynamical systems. In Section 3, we transform (1.1) into a continuous stochastic dynamical system. Section 4 is devoted to the uniform estimates of solutions. These estimates are necessary for proving the existence of bounded absorbing sets and the asymptotic compactness of the solution for (1.1). In the last section, we establish the asymptotic compactness of the solution operator by giving uniform estimates on the tail of solutions and then prove the existence of the random attractor.

We denote by $\|\cdot\|$ and $(\cdot, \cdot)$ the norm and the inner product in $H=L^{2}\left(\mathbb{R}^{2}\right)$ respectively, and we use $\|\cdot\|_{L^{p}}$ to denote the norm in $L^{p}\left(\mathbb{R}^{2}\right) . H^{k}\left(\mathbb{R}^{2}\right), k \in Z^{+}$are the usual Sobolev spaces. Moreover, the norm of a general Banach space $X$ is written as $\|\cdot\|_{X}$. The letters $c$ and $c_{i}(i=1,2, \cdots)$ are generic positive constants which may change their values from line to line or even in the same line.

\section{Preliminaries}

We recall some basic concepts related to random attractors for stochastic dynamical systems. For additional details, the reader is referred to $[5,6]$. Let $\left(X,\|\cdot\|_{X}\right)$ be a separable Hilbert space with Borel $\sigma$-algebra $\mathscr{B}(X)$ endowed with the distance $d$, and let $(\Omega, \mathcal{F}, \mathbb{P})$ be a probability space. We also denote the mappings $S(t, s ; \omega): X \rightarrow X,-\infty<s \leq t<\infty$ with explicit dependence on $\omega$. In most applica- 
tions there exists a group $\theta_{t}, t \in \mathbb{R}$, of measure preserving transformations of $(\Omega, \mathcal{F}, \mathbb{P})$ with the property that for all $s<t$ and $x \in X$ we have

$$
S(t, s ; \omega) x=S\left(t-s, 0 ; \theta_{s} \omega\right) x, \quad P \text { - a.e. }
$$

In applications to stochastic evolution equations driven by white noise, $\omega(t)$ is the two-sided Wiener space $C_{0}(\mathbb{R} ; X)$ of continuous functions with values in a Banach space $X$, equal to 0 at $t=0$. In this case $\theta_{t}$ is defined as

$$
\left(\theta_{t} \omega\right)(s)=\omega(t+s)-\omega(t), \quad s, t \in \mathbb{R} .
$$

Definition 2.1. Let $t \in \mathbb{R}$ and $\omega \in \Omega$. A stochastic dynamical system with time $t$ on a complete and separable metric space $(X, d)$ with Borel $\sigma$-algebra $\mathscr{B}$ over $\left\{\theta_{t}\right\}$ on $(\Omega, \mathcal{F}, \mathbb{P})$ is a measurable map

$$
S(t, s ; \omega): X \rightarrow X, \quad-\infty<s \leq t<\infty
$$

such that $S(s, s ; \omega) x=x$ and $S(t, s ; \omega) x=S(t, r ; \omega) S(r, s ; \omega) x$ for all $s \leq r \leq t \in \mathbb{R}$ and for all $x \in X, \omega \in \Omega$.

Definition 2.2. Given $t \in \mathbb{R}$ and $\omega \in \Omega, K(t, \omega) \subset X$ is called an attracting set if for all bounded sets $B \subset X$,

$$
d(S(t, s ; \omega) B, K(t, \omega)) \rightarrow 0, \quad s \rightarrow-\infty,
$$

where $d(A, B)$ is the semidistance defined by

$$
d(A, B)=\sup _{x \in A} \inf _{y \in B} d(x, y) .
$$

Definition 2.3. A family $A(\omega)(\omega \in \Omega)$ of the closed subsets of $X$ is measurable, if for all $x \in X$, the mapping $\omega \mapsto d(A(\omega), x)$ is measurable.

DeFinition 2.4. Define the random omega limit set of a bounded set $B \subset X$ at time $t$ as

$$
A(B, t ; \omega)=\bigcap_{T<t s<T} \overline{\bigcup_{s<T} S(t, s ; \omega) B}
$$

Definition 2.5. Let $S(t, s ; \omega)_{t \geq s, \omega \in \Omega}$ be a stochastic dynamical system, and let $A(\omega)$ be a stochastic set such that

(1) $A(\omega)$ is the minimal closed set such that for $t \in \mathbb{R}, B \subset X$,

$$
d(S(t, s ; \omega) B, A(\omega)) \rightarrow 0, \quad s \rightarrow-\infty,
$$

here we call $A(\omega)$ the attractor of $B$ ( $B$ is a deterministic set);

(2) $A(\omega)$ is the largest compact measurable set, which is invariant in sense that

$$
S(t, s ; \omega) A\left(\theta_{s} \omega\right)=A\left(\theta_{t} \omega\right), \quad s \leq t .
$$

Then $A(\omega)$ is said to be the random attractor. 
TheOREm 2.6. ([5, 6]) Let $S(t, s ; \omega)_{t \geq s, \omega \in \Omega}$ be a stochastic dynamical system satisfying the following conditions:

(i) $S(t, r ; \omega) S(r, s ; \omega) x=S(t, s ; \omega) x$ for all $s \leq r \leq t$ and $x \in X$,

(ii) $S(t, s ; \omega)$ is continuous in $X$, for all $s \leq t$,

(iii) for all $s<t$ and $x \in X$, the mapping $\omega \mapsto S(t, s ; \omega) x$ is measurable from $(\Omega, \mathcal{F}, \mathbb{P})$ to $(X, \mathscr{B}(X))$,

(iv) for all $t \in \mathbb{R}, x \in X$ and $P$-a.e. $\omega$, the mapping $s \mapsto S(t, s ; \omega) x$ is right continuous at any point.

Assume that there exists a group $\theta_{t}, t \in \mathbb{R}$ of measure preserving mappings such that

$$
S(t, s ; \omega) x=S\left(t-s, 0 ; \theta_{s} \omega\right) x, \quad P-a . e .
$$

holds and for $P$-a.e. there exists a compact attracting set $K(\omega)$ at time 0 . For P-a.e. $\omega \in \Omega$, we set $\Lambda(\omega)=\overline{\bigcup_{B \subset X} A(B, \omega)}$, where the union is taken over all the bounded subsets of $X$ and $A(B, \omega)$ is given by

$$
A(B, 0 ; \omega)=\bigcap_{T<0} \overline{\bigcup_{s<T} S(0, s ; \omega) B} .
$$

Then $\Lambda(\omega)$ is the random attractor.

\section{The hydrodynamical equations with additive noise}

In the entire space $\mathbb{R}^{2}$ there is a continuous stochastic dynamical system $\left(S(t, s ; \omega) ; H^{1}\left(\mathbb{R}^{2}\right)\right)$ generated by the stochastic hydrodynamical equation defined with additive noise:

$$
d u+(\lambda u-\Delta u-u \times \Delta u) d t=\sum_{i=1}^{m} \phi_{i}(x) d w_{i}(t), \quad x \in \mathbb{R}^{2},
$$

with the initial condition

$$
u(x, s)=u_{s}(x),
$$

where $\lambda$ is a positive constant, $\phi_{i}(x)(i=1,2, \cdots, m)$ are given smooth functions defined on $\mathbb{R}^{2}$, and $\left\{w_{i}\right\}_{i=1}^{m}$ are independent two-sided real-valued Wiener processes on a probability space $\left(\Omega, \mathcal{F}, \mathbb{P} ; \theta_{t}\right)$. For the deterministic case, it is obvious that there is a dynamical system $\left(S(t), H^{1}\left(\mathbb{R}^{2}\right)\right)$; see $[7,10]$. For the stochastic equation, there is a continuous stochastic dynamical system $\left(S(t, s ; \omega) ; H^{1}\left(\mathbb{R}^{2}\right)\right)$ generated by the stochastic hydrodynamical equation defined with additive noise; see $[8,9,11,14]$. Now we need to convert the stochastic equation with a random additive term into a deterministic equation with a random parameter.

Let $\alpha>0$ be given; we shall impose a condition on $\alpha$ below. Given $i=1, \ldots, m$, let $z_{i}$ be the stationary (ergodic) solution of the one-dimensional Ornstein-Uhlenbech equation

$$
d z_{i}+\alpha z_{i} d t=d w_{i}(t)
$$

so that

$$
z_{i}(t)=\int_{-\infty}^{t} e^{-\alpha(t-s)} d w_{i}(s)
$$


Putting $z(t)=\sum_{i=1}^{m} \phi_{i}(x) z_{i}(t)$ we have

$$
d z+\alpha z d t=\sum_{i=1}^{m} \phi_{i}(x) d w_{i}(t)
$$

Then the trajectories of $z_{i}$ are $P$-a.e. continuous. Let $\phi_{i}(x) \in H^{3}\left(\mathbb{R}^{2}\right)$. We know that for all $\varepsilon>0$ one can choose $\alpha>0$ such that

$$
\mathbb{E}\left|z_{1}(0)\right|^{2} \leq \varepsilon
$$

This is possible since $0 \leq \mathbb{E}\left|z_{1}(0)\right|^{2}=\operatorname{Var}\left(z_{1}(0)\right)$, and $\operatorname{Var}\left(z_{1}(0)\right) \rightarrow 0$ as $\alpha \rightarrow \infty$; see [7]. From (3.5) and the ergodic theorem we obtain

$$
\lim _{s \rightarrow-\infty}-\frac{1}{s} \int_{s}^{0} \sum_{i=1}^{m}\left|z_{i}(t)\right|^{2} d t=m \mathbb{E}\left|z_{1}(0)\right|^{2} \rightarrow 0 \quad P \text {-a.e. }
$$

as $\alpha \rightarrow \infty$. In addition, for $P$-a.e. $\omega \in \Omega$, we have that

$$
\sum_{i=1}^{m}\left(\left|z_{i}(t)\right|^{2}+\left|z_{i}(t)\right|^{p}\right)
$$

grows at most polynomially as $t \rightarrow-\infty$, where $p \geq 2$.

Let $u(t)=v(t)+z(t)$. We have

$$
\frac{d v}{d t}+\lambda v-\Delta v=\Delta z+(\alpha-\lambda) z+(v+z) \times \Delta(v+z),
$$

with initial condition

$$
v_{s}(x)=u_{s}(x)-z(s) .
$$

We now consider the properties of (3.8)-(3.9).

\section{Uniform estimates of solutions}

In this section we consider the uniform estimates of solutions for (3.8)-(3.9) to derive uniform estimates on the solution of (3.1)-(3.2) defined on $\mathbb{R}^{2}$ at $t=0$, for the purpose of proving the existence of a bounded random absorbing set and the asymptotic compactness of the stochastic dynamical system associated with the equation. In particular, we will show that the tails of the solution, i.e., solutions evaluated at large values of $|x|$, are uniformly small when $t \rightarrow 0^{-}$. Now we consider the equation

$$
d v+(\lambda v-\Delta v) d t=\Delta z d t+(\alpha-\lambda) z d t+((v+z) \times \Delta(v+z)) d t .
$$

Lemma 4.1. Let $\phi_{i}(x) \in H^{3}\left(\mathbb{R}^{2}\right)$, and $v(t)$ be the solution of (3.8)-(3.9). For any given $\eta>0$ and $u_{s} \in H$ satisfying $\left\|u_{s}\right\| \leq \eta$, there exist random radii $r_{0}(\omega), r_{1}(\omega), r_{2}(\omega), r_{3}(\omega)$ and $s_{1}(\omega) \leq-1$, such that for all $s \leq s_{1}(\omega)$ the following inequalities hold $P$-a.e.:

$$
\begin{aligned}
& \|v(t)\|^{2} \leq r_{0}(\omega),\|u(t)\|^{2} \leq r_{1}(\omega), \quad t \in[-1,0], \\
& \int_{-1}^{0}\|\nabla v(t)\|^{2} d t \leq r_{2}(\omega), \\
& \int_{-1}^{0}\|\nabla v(t)+\nabla z(t)\|^{2} d t \leq r_{3}(\omega) .
\end{aligned}
$$


Proof. Taking the inner product of Equation (3.8) with $v$ in $H$, we can obtain

$$
\begin{aligned}
\frac{1}{2} \frac{d}{d t}\|v\|^{2}+\lambda\|v\|^{2}+\|\nabla v\|^{2} & =(\Delta z, v)+((\alpha-\lambda) z, v)+((v+z) \times \Delta(v+z), v) \\
& =(\Delta z+(\alpha-\lambda) z, v)+(z \times \Delta v, v)+(z \times \Delta z, v),
\end{aligned}
$$

because $(a \times b) \cdot a=(a \times b) \cdot b=0$ for any $a, b, c \in \mathbb{R}^{3}$. Moreover Young's inequality implies that

$$
|(\Delta z+(\alpha-\lambda) z, v)| \leq \frac{\lambda}{8}\|v\|^{2}+c\left(\|\Delta z\|^{2}+\|z\|^{2}\right) \leq \frac{\lambda}{8}\|v\|^{2}+c_{1}\|z\|_{H^{2}}^{2} .
$$

For the third term on the right hand side of (4.2), we have the estimate

$$
|(z \times \Delta z, v)| \leq\|v\|\|z \times \Delta z\| \leq \frac{\lambda}{8}\|v\|^{2}+c\|z\|_{L^{\infty}}^{2}\|\Delta z\|^{2} \leq \frac{\lambda}{8}\|v\|^{2}+c_{2}\|z\|_{H^{2}}^{4},
$$

where the last inequality is due to the Sobolev embedding theorem $H^{2}\left(\mathbb{R}^{2}\right) \hookrightarrow L^{\infty}\left(\mathbb{R}^{2}\right)$.

We let $\|\nabla v\|_{L^{\infty}} \leq b\|v\|_{H^{3}}$, where $b$ is a positive constant. For the second term we have the estimate

$$
\begin{aligned}
|(z \times \Delta v, v)| & =|(\nabla z \times \nabla v, v)| \leq \int_{\mathbb{R}^{2}}|v\|\nabla v\| \nabla z| d x \leq\|v\|\|\nabla v\|\|\nabla z\|_{L^{\infty}} \\
& \leq \frac{1}{4}\|\nabla v\|^{2}+\|\nabla z\|_{L^{\infty}}^{2}\|v\|^{2} \leq \frac{1}{4}\|\nabla v\|^{2}+b^{2}\|z\|_{H^{3}}^{2}\|v\|^{2} .
\end{aligned}
$$

By using the above estimates we can obtain the inequality

$$
\frac{d}{d t}\|v\|^{2}+\left(\lambda-2 b^{2}\|z\|_{H^{3}}^{2}\right)\|v\|^{2}+\frac{\lambda}{2}\|v\|^{2}+\|\nabla v\|^{2} \leq c_{4}\left(\|z\|_{H^{2}}^{2}+\|z\|_{H^{2}}^{4}\right) .
$$

We let $G(t)=c_{4}\left(\|z\|_{H^{2}}^{2}+\|z\|_{H^{2}}^{4}\right) \leq c \sum_{k=1}^{m}\left(\left|z_{k}\right|^{2}+\left|z_{k}\right|^{4}\right)$, which grows at most polynomially as $t \rightarrow-\infty P$-a.e., since $\sum_{k=1}^{m}\left(\left|z_{k}\right|^{2}+\left|z_{k}\right|^{4}\right)$ grows at most polynomially as $t \rightarrow-\infty P$-a.e. [7]. Then we have

$$
\frac{d}{d t}\|v\|^{2}+\left(\lambda-2 b^{2}\|z\|_{H^{3}}^{2}\right)\|v\|^{2}+\frac{\lambda}{2}\|v\|^{2}+\|\nabla v\|^{2} \leq G(t) .
$$

By Gronwall's inequality for $s \leq-1$ and $t \in[-1,0]$, we have

$$
\begin{aligned}
& \|v(t)\|^{2} \\
& \leq e^{-\int_{s}^{t}\left(\lambda-2 b^{2}\|z(\sigma)\|_{H^{3}}^{2}\right) d \sigma}\|v(s)\|^{2}+\int_{s}^{t} e^{-\int_{\tau}^{t}\left(\lambda-2 b^{2}\|z(\sigma)\|_{H^{3}}^{2}\right) d \sigma} G(\tau) d \tau \\
& \leq e^{\lambda} e^{-s\left(-\lambda-\frac{2 b^{2}}{s} \int_{s}^{0}\|z(\sigma)\|_{H^{3}}^{2} d \sigma\right)}\|v(s)\|^{2}+e^{\lambda} \int_{s}^{0} e^{-\tau\left(-\lambda-\frac{2 b^{2}}{\tau} \int_{\tau}^{0}\|z(\sigma)\|_{H^{3}}^{2} d \sigma\right)} G(\tau) d \tau .
\end{aligned}
$$

From the ergodic theorem in [5], the process $\|z\|_{H^{3}}^{2}$ is stationary and ergodic, thus there exist $s_{0}(\omega)$ such that for any $s<s_{0}(\omega)$, we have

$$
-\lambda-\frac{2 b^{2}}{s} \int_{s}^{0}\|z(\sigma)\|_{H^{3}}^{2} d \sigma \leq-\lambda-\frac{2 b^{2}}{s} \int_{s}^{0} \sum_{k=1}^{m}\left|z_{k}(\sigma)\right|^{2} d \sigma \leq-\lambda+\frac{\lambda}{2}=\frac{-\lambda}{2} .
$$


Therefore we see that

$$
\begin{aligned}
e^{-\int_{s}^{t}\left(\lambda-2 b^{2}\|z(\sigma)\|_{H^{3}}^{2}\right) d \sigma} & \leq e^{\lambda} e^{-s\left(-\lambda-\frac{2 b^{2}}{s} \int_{s}^{0}\|z(\sigma)\|_{H^{3}}^{2} d \sigma\right)} \\
& \leq e^{\lambda} e^{-s\left(-\lambda-\frac{2 b^{2}}{s} \int_{s}^{0} \sum_{k=1}^{m}\left|z_{k}(\sigma)\right|^{2} d \sigma\right)} \leq e^{\lambda} e^{\frac{\lambda s}{2}}
\end{aligned}
$$

decays exponentially as $s \rightarrow-\infty$. From (4.4) we have

$$
\|v(t)\|^{2} \leq e^{\lambda} e^{\frac{\lambda s}{2}}\|v(s)\|^{2}+e^{\lambda} \int_{-\infty}^{0} e^{-\tau\left(-\lambda-\frac{2 b^{2}}{\tau} \int_{\tau}^{0}\|z(\sigma)\|_{H^{3}}^{2} d \sigma\right)} G(\tau) d \tau .
$$

Because $G(\tau)$ is multiplied by a function which decays exponentially, the integral in (4.6) converges. Given $\eta>0$ we can choose $s_{1}(\omega)<s_{0}(\omega)$ depending only on $\omega$ such that

$$
e^{\frac{\lambda s}{2}} \eta^{2} \leq 1, \quad \forall s \leq s_{1}(\omega) .
$$

We can then deduce from (4.6) that for all $s \leq \min \left\{-1, s_{1}(\omega)\right\}, t \in[-1,0]$,

$$
\begin{aligned}
\|v(t)\|^{2} \leq & r_{0}(\omega):=2 e^{\lambda}\left(1+\sup _{s \leq-1} e^{\frac{\lambda s}{2}}\|z(s)\|^{2}\right) \\
& +e^{\lambda} \int_{-\infty}^{0} e^{-\tau\left(-\lambda-\frac{2 b^{2}}{\tau} \int_{\tau}^{0}\|z(\sigma)\|_{H^{3}}^{2} d \sigma\right)} G(\tau) d \tau .
\end{aligned}
$$

Similarly, since $z(s)$ grows at most polynomially as $s \rightarrow-\infty$ and $z(s)$ is multiplied by a function which decays exponentially, then the term

$$
\sup _{s \leq-1} e^{\frac{\lambda s}{2}}\|z(s)\|^{2}
$$

is bounded. Now we can integrate (4.3) on $[-1,0]$ and obtain

$$
\int_{-1}^{0}\|\nabla v(t)\|^{2} d t \leq r_{0}(\omega)+\int_{-1}^{0} G(\tau) d \tau+c r_{0}(\omega) \int_{-1}^{0}\|z(\tau)\|_{H^{3}}^{2} d \tau:=r_{2}(\omega)
$$

and

$$
\int_{-1}^{0}\|\nabla v(t)+\nabla z\|^{2} d t \leq 2 r_{2}(\omega)+2 \int_{-1}^{0}\|\nabla z\|^{2} d t:=r_{3}(\omega) .
$$

On the other hand, we can obtain

$$
\|u(t)\|^{2} \leq 2\|v(t)\|^{2}+2\|z(t)\|^{2} \leq 2 r_{0}(\omega)+2 \sup _{0 \leq t \leq-1}\|z(t)\|^{2}:=r_{1}(\omega)
$$

The proof is complete.

Lemma 4.2. Let $\phi_{i}(x) \in H^{3}\left(\mathbb{R}^{2}\right)$ and $v(t)$ be the solution of (3.8)-(3.9). For any given $\eta>0$ and $u_{s} \in H$ satisfying $\left\|u_{s}\right\| \leq \eta$, there exist random radii $r_{4}(\omega), r_{5}(\omega)$ such that the following inequalities hold $P$-a.e.:

$$
\begin{aligned}
& \int_{-\infty}^{0} e^{-\tau\left(-\lambda-\frac{2 b^{2}}{\tau} \int_{\tau}^{0}\|z(\sigma)\|_{H^{3}}^{2} d \sigma\right)}\|v(\tau)\|^{2} d \tau \leq r_{4}(\omega), \\
& \int_{-\infty}^{0} e^{-\tau\left(-\lambda-\frac{2 b^{2}}{\tau} \int_{\tau}^{0}\|z(\sigma)\|_{H^{3}}^{2} d \sigma\right)}\|\nabla v(\tau)\|^{2} d \tau \leq r_{5}(\omega) .
\end{aligned}
$$


Proof. Integrating from $s<-1$ to 0 on the both sides of (4.3), we have

$$
\begin{gathered}
\|v(0)\|^{2}+\frac{\lambda}{2} \int_{s}^{0} e^{-\tau\left(-\lambda-\frac{2 b^{2}}{\tau} \int_{\tau}^{0}\|z(\sigma)\|_{H^{3}}^{2} d \sigma\right)}\|v(\tau)\|^{2} d \tau \\
\quad+\int_{s}^{0} e^{-\tau\left(-\lambda-\frac{2 b^{2}}{\tau} \int_{\tau}^{0}\|z(\sigma)\|_{H^{3}}^{2} d \sigma\right)}\|\nabla v(\tau)\|^{2} d \tau \\
\leq e^{-s\left(-\lambda-\frac{2 b^{2}}{s} \int_{s}^{0}\|z(\sigma)\|_{H^{3}}^{2} d \sigma\right)}\|v(s)\|^{2}+\int_{s}^{0} e^{-\tau\left(-\lambda-\frac{2 b^{2}}{\tau} \int_{\tau}^{0}\|z(\sigma)\|_{H^{3}}^{2} d \sigma\right)} G(\tau) d \tau .
\end{gathered}
$$

For the first term of the right hand side of (4.10), there exists a $s_{2}(\omega) \leq s_{1}(\omega)$ satisfying

$$
e^{-s\left(-\lambda-\frac{2 b^{2}}{s} \int_{s}^{0}\|z(\sigma)\|_{H^{3}}^{2} d \sigma\right)}\|v(s)\|^{2} \leq e^{\frac{\lambda s}{2}} \eta^{2} \leq 1 .
$$

For the second term of the right hand side of (4.10), since $G(\tau)$ grows at most polynomially as $\tau \rightarrow-\infty P$-a.e. and is multiplied by a function which decays exponentially, the integral in (4.10) converges. From (4.10) we can then deduce the results. The proof is complete.

Lemma 4.3. Let $\phi_{i}(x) \in H^{3}\left(\mathbb{R}^{2}\right)$ and $v(t)$ be the solution of (3.8)-(3.9). For any given $\eta>0$ and $u_{s} \in H$ satisfying $\left\|u_{s}\right\| \leq \eta$, there exist random radii $r_{6}(\omega), r_{7}(\omega), r_{8}(\omega)$ and $s_{1}(\omega) \leq-1$, such that for all $s \leq s_{1}(\omega)$, the following inequalities hold $P$-a.e.

$$
\begin{aligned}
& \|\nabla v(t)\|^{2} \leq r_{6}(\omega),\|\nabla u(t)\|^{2} \leq r_{7}(\omega), \quad t \in[-1,0], \\
& \int_{-1}^{0}\|\Delta v(t)\|^{2} d t \leq r_{8}(\omega) .
\end{aligned}
$$

Proof. Taking the inner product of Equation (3.8) with $-\Delta v$ in $H$, we obtain

$$
\begin{aligned}
& \frac{1}{2} \frac{d}{d t}\|\nabla v\|^{2}+\lambda\|\nabla v\|^{2}+\|\Delta v\|^{2} \\
= & (\Delta z,-\Delta v)+((\alpha-\lambda) z,-\Delta v)+((v+z) \times \Delta(v+z),-\Delta v) \\
= & (\Delta z+(\alpha-\lambda) z,-\Delta v)+(v \times \Delta z,-\Delta v)+(z \times \Delta z,-\Delta v) .
\end{aligned}
$$

Moreover, Young's inequality implies that

$$
|(\Delta z+(\alpha-\lambda) z,-\Delta v)| \leq \frac{1}{8}\|\Delta v\|^{2}+c\left(\|\Delta z\|^{2}+\|z\|^{2}\right) \leq \frac{1}{8}\|\Delta v\|^{2}+c_{6}\|z\|_{H^{2}}^{2} .
$$

For the third term on the right hand side of (4.3), we have the following estimate

$$
|(z \times \Delta z,-\Delta v)| \leq\|\Delta v\|\|z \times \Delta z\| \leq \frac{1}{8}\|\Delta v\|^{2}+c\|z\|_{L^{\infty}}^{2}\|\Delta z\|^{2} \leq \frac{1}{8}\|\Delta v\|^{2}+c_{7}\|z\|_{H^{2}}^{4} .
$$

For the second term we have the estimate

$$
\begin{gathered}
|(v \times \Delta z,-\Delta v)| \leq\|\Delta v\|\|v\|_{L^{4}}\|\Delta z\|_{L^{4}} \leq \frac{1}{4}\|\Delta v\|^{2}+c\|v\|_{L^{4}}^{2}\|\Delta z\|_{L^{4}}^{2} \\
\leq \frac{1}{4}\|\Delta v\|^{2}+c\|v\|\|\nabla v\|\|\Delta z\|\|\nabla \Delta z\| \leq \frac{1}{4}\|\Delta v\|^{2}+c_{8}\|v\|^{2}\|\nabla v\|^{2}+c_{9}\|z\|_{H^{2}}^{2}\|z\|_{H^{3}}^{2},
\end{gathered}
$$

where we use the $\varepsilon$-Young's inequality and the Gagliardo-Nirenberg inequality:

$$
\|v\|_{L^{4}} \leq c\|v\|^{\frac{1}{2}}\|\nabla v\|^{\frac{1}{2}} .
$$


Using the above estimates we obtain the inequality

$$
\frac{d}{d t}\|\nabla v\|^{2}+\lambda\|\nabla v\|^{2}+\|\Delta v\|^{2} \leq 2 c_{8}\|v\|^{2}\|\nabla v\|^{2}+F(t),
$$

where $F(t)=c_{10}\left(\|z\|_{H^{2}}^{2}+\|z\|_{H^{2}}^{4}+\|z\|_{H^{2}}^{2}\|z\|_{H^{3}}^{2}\right) \leq c \sum_{k=1}^{m}\left(\left|z_{k}\right|^{2}+\left|z_{k}\right|^{4}\right)$, which grows at most polynomially as $t \rightarrow-\infty \mathrm{P}$-a.e.

We then have

$$
\frac{d}{d t}\|\nabla v\|^{2}-2 c_{8}\|v\|^{2}\|\nabla v\|^{2} \leq F(t)
$$

Integrating from $\theta$ to $t$ for any $-1 \leq \theta \leq t \leq 0$, we have

$$
\begin{aligned}
\|\nabla v(t)\|^{2} & \leq e^{\int_{\theta}^{t} 2 c_{8}\|v(\sigma)\|^{2} d \sigma}\|\nabla v(\theta)\|^{2}+\int_{\theta}^{t} e^{\int_{\tau}^{t} 2 c_{8}\|v(\sigma)\|^{2} d \sigma} F(\tau) d \tau \\
& \leq\left(\|\nabla v(\theta)\|^{2}+\int_{-1}^{0} F(\tau) d \tau\right) e^{\int_{-1}^{0} 2 c_{8}\|v(\sigma)\|^{2} d \sigma} .
\end{aligned}
$$

Now integrating with respect to $\theta$ on $[-1,0]$ on both sides of $(4.14)$, then there exists $s_{1}(\omega)$ as in Lemma 4.1 , such that for all $s<s_{1}(\omega)$ we have

$$
\begin{aligned}
\|\nabla v(t)\|^{2} & \leq\left(\int_{-1}^{0}\|\nabla v(\theta)\|^{2} d \theta+\int_{-1}^{0} F(\tau) d \tau\right) e^{\int_{-1}^{0} 2 c_{8}\|v(\sigma)\|^{2} d \sigma} \\
& \leq\left(r_{2}(\omega)+\int_{-1}^{0} F(\tau) d \tau\right) e^{2 c_{8} r_{0}(\omega)}:=r_{6}(\omega) .
\end{aligned}
$$

Now we integrate with respect to $t$ on $[-1,0]$ on the both sides of $(4.12)$ and deduce that

$$
\int_{-1}^{0}\|\Delta v\|^{2} d s \leq r_{8}(\omega)
$$

On the other hand, we can obtain

$$
\|\nabla u(t)\|^{2} \leq 2\|\nabla v(t)\|^{2}+2\|\nabla z(t)\|^{2} \leq 2 r_{6}(\omega)+2 \sup _{0 \leq t \leq-1}\|\nabla z(t)\|^{2}:=r_{7}(\omega) .
$$

The proof is complete.

Lemma 4.4. Let $\phi_{i}(x) \in H^{3}\left(\mathbb{R}^{2}\right), \eta>0$ be given and $u_{s} \in H$ satisfy $\left\|u_{s}\right\| \leq \eta$. Then for every $\varepsilon>0$ and $P$-a.e. $\omega \in \Omega$, there exist $s^{*}(\omega, \varepsilon) \leq-1$ and $R^{*}(\omega, \varepsilon)>0$, such that for all $s \leq s^{*}(\omega, \varepsilon)$ and $R>R^{*}(\omega, \varepsilon)$, the solution $v(t)$ of (4.2) with $v_{s}=u_{s}-z(s)$ satisfies

$$
\int_{|x| \geq R^{*}}|v(t)|^{2} d x \leq \varepsilon, \quad t \in[-1,0] .
$$

Proof. Let $\rho(s)$ be a smooth function defined on $\mathbb{R}^{+}$such that $0 \leq \rho \leq 1$ for all $s \in \mathbb{R}^{+}$, and

$$
\rho(s)= \begin{cases}0, & \text { for } \quad 0 \leq s \leq 1 \\ 1, & \text { for } s \geq 2 .\end{cases}
$$


Then there exists a positive constant $c$ such that $\left|\rho^{\prime}(s)\right| \leq c$ for all $s \in \mathbb{R}^{+}$. Taking the inner product of Equation (4.2) with $\rho\left(\frac{|x|^{2}}{k^{2}}\right) v$ in $H$, we obtain

$$
\begin{aligned}
& \frac{1}{2} \frac{d}{d t} \int_{\mathbb{R}^{2}} \rho|v|^{2} d x+\lambda \int_{\mathbb{R}^{2}} \rho|v|^{2} d x+\left(-\Delta v, \rho\left(\frac{|x|^{2}}{k^{2}}\right) v\right) \\
= & \left(\Delta z+(\alpha-\lambda) z, \rho\left(\frac{|x|^{2}}{k^{2}}\right) v\right)+\left((v+z) \times \Delta(v+z), \rho\left(\frac{|x|^{2}}{k^{2}}\right) v\right) .
\end{aligned}
$$

Noting that

$$
\left(-\Delta v, \rho\left(\frac{|x|^{2}}{k^{2}}\right) v\right)=\left(\nabla v, \nabla\left(\rho\left(\frac{|x|^{2}}{k^{2}}\right) v\right)\right)=\int_{\mathbb{R}^{2}} \rho|\nabla v|^{2} d x+\left(\nabla v, v \rho^{\prime}(s) \frac{2 x}{k^{2}}\right),
$$

from (4.16) we have

$$
\begin{aligned}
& \frac{1}{2} \frac{d}{d t} \int_{\mathbb{R}^{2}} \rho|v|^{2} d x+\lambda \int_{\mathbb{R}^{2}} \rho|v|^{2} d x+\int_{\mathbb{R}^{2}} \rho|\nabla v|^{2} d x \\
=- & \left(\nabla v, v \rho^{\prime}(s) \frac{2 x}{k^{2}}\right)+\left(\Delta z+(\alpha-\lambda) z, \rho\left(\frac{|x|^{2}}{k^{2}}\right) v\right) \\
& +\left((v+z) \times \Delta(v+z), \rho\left(\frac{|x|^{2}}{k^{2}}\right) v\right) .
\end{aligned}
$$

Now we estimate the first term of the right side of (4.17):

$$
\left|-\left(\nabla v, v \rho^{\prime}(s) \frac{2 x}{k^{2}}\right)\right| \leq \frac{2 \sqrt{2}}{k} \int_{k \leq|x| \leq \sqrt{2} k}\left|v \| \rho^{\prime}(s)\right||\nabla v| d x \leq \frac{c}{k}\left(\|v\|^{2}+\|\nabla v\|^{2}\right) .
$$

The second term of the right hand side of (4.17) is bounded by

$$
\left|\left(\Delta z+(\alpha-\lambda) z, \rho\left(\frac{|x|^{2}}{k^{2}}\right) v\right)\right| \leq \frac{\lambda}{8} \int_{\mathbb{R}^{2}} \rho|v|^{2} d x+c \int_{\mathbb{R}^{2}} \rho\left(|\Delta z|^{2}+|z|^{2}\right) d x .
$$

For the third term on the right hand side of (4.17), we have

$$
\left((v+z) \times \Delta(v+z), \rho\left(\frac{|x|^{2}}{k^{2}}\right) v\right)=\left(z \times \Delta v, \rho\left(\frac{|x|^{2}}{k^{2}}\right) v\right)+\left(z \times \Delta z, \rho\left(\frac{|x|^{2}}{k^{2}}\right) v\right)
$$

The second term of the right hand side of (4.18) is bounded by

$$
\begin{aligned}
& \left|\left(z \times \Delta z, \rho\left(\frac{|x|^{2}}{k^{2}}\right) v\right)\right| \leq \frac{\lambda}{8} \int_{\mathbb{R}^{2}} \rho|v|^{2} d x+\int_{\mathbb{R}^{2}} \rho|z|^{2}|\Delta z|^{2} d x \\
\leq & \frac{\lambda}{8} \int_{\mathbb{R}^{2}} \rho|v|^{2} d x+c\|z\|_{L^{\infty}}^{2} \int_{\mathbb{R}^{2}} \rho|\Delta z|^{2} d x \leq \frac{\lambda}{8} \int_{\mathbb{R}^{2}} \rho|v|^{2} d x+c\|z\|_{H^{2}}^{2} \int_{\mathbb{R}^{2}} \rho|\Delta z|^{2} d x .
\end{aligned}
$$

For the first term of the right hand side of (4.18) we have the estimate

$$
\begin{aligned}
& \left|\left(z \times \Delta v, \rho\left(\frac{|x|^{2}}{k^{2}}\right) v\right)\right| \leq\left|\left(\nabla z \times \nabla v, \rho\left(\frac{|x|^{2}}{k^{2}}\right) v\right)\right|+\left|\left(z \times \nabla v, v \rho^{\prime}(s) \frac{2 x}{k^{2}}\right)\right| \\
\leq & \frac{1}{4} \int_{\mathbb{R}^{2}} \rho|\nabla v|^{2} d x+b^{2}\|z\|_{H^{3}}^{2} \int_{\mathbb{R}^{2}} \rho|v|^{2} d x+\frac{c}{k}\left(\|\nabla v\|^{2}+\|z\|_{H^{2}}^{2}\|v\|^{2}\right) .
\end{aligned}
$$


Using the above estimates we obtain the inequality

$$
\begin{aligned}
& \frac{d}{d t} \int_{\mathbb{R}^{2}} \rho|v|^{2} d x+\left(\lambda-2 b^{2}\|z\|_{H^{3}}^{2}\right) \int_{\mathbb{R}^{2}} \rho|v|^{2} d x+\int_{\mathbb{R}^{2}} \rho|\nabla v|^{2} d x \\
\leq & \frac{c_{12}}{k}\left(\|v\|^{2}+\|\nabla v\|^{2}\right)+\frac{c_{13}}{k}\|z\|_{H^{2}}^{2}\|v\|^{2} \\
& +c_{14}\left(\|z\|_{H^{2}}^{2} \int_{\mathbb{R}^{2}} \rho|\Delta z|^{2} d x+\int_{\mathbb{R}^{2}} \rho\left(|\Delta z|^{2}+|z|^{2}\right) d x\right) .
\end{aligned}
$$

We let $E(t)=c_{14}\left(\|z\|_{H^{2}}^{2} \int_{\mathbb{R}^{2}} \rho|\Delta z|^{2} d x+\int_{\mathbb{R}^{2}} \rho\left(|\Delta z|^{2}+|z|^{2}\right) d x\right)$ : It is easy to see that $E(t) \leq c \sum_{k=1}^{m}\left(\left|z_{k}\right|^{2}+\left|z_{k}\right|^{4}\right)$, which grows at most polynomially as $t \rightarrow-\infty P$-a.e. Then we have

$$
\begin{aligned}
& \frac{d}{d t} \int_{\mathbb{R}^{2}} \rho|v|^{2} d x+\left(\lambda-2 b^{2}\|z\|_{H^{3}}^{2}\right) \int_{\mathbb{R}^{2}} \rho|v|^{2} d x \\
\leq & \frac{c_{12}}{k}\left(\|v\|^{2}+\|\nabla v\|^{2}\right)+\frac{c_{13}}{k}\|z\|_{H^{2}}^{2}\|v\|^{2}+E(t) .
\end{aligned}
$$

By Gronwall's inequality for $s \leq-1$ and $t \in[-1,0]$, we have

$$
\begin{aligned}
& \int_{\mathbb{R}^{2}} \rho|v(t)|^{2} d x \\
\leq & e^{-\int_{s}^{t}\left(\lambda-2 b^{2}\|z(\sigma)\|_{H^{3}}^{2}\right) d \sigma} \int_{\mathbb{R}^{2}} \rho|v(s)|^{2} d x \\
& +\int_{s}^{t} e^{-\int_{\tau}^{t}\left(\lambda-2 b^{2}\|z(\sigma)\|_{H^{3}}^{2}\right) d \sigma}\left[\frac{c_{12}}{k}\left(\|v\|^{2}+\|\nabla v\|^{2}\right)+\frac{c_{13}}{k}\|z\|_{H^{2}}^{2}\|v\|^{2}+E(\tau)\right] d \tau \\
\leq & e^{\lambda} e^{-s\left(-\lambda-\frac{2 b^{2}}{s} \int_{s}^{0}\|z(\sigma)\|_{H^{3}}^{2} d \sigma\right)\|v(s)\|^{2}} \\
& +e^{\lambda} \frac{c}{k} \int_{-\infty}^{0} e^{-\tau\left(-\lambda-\frac{2 b^{2}}{\tau} \int_{\tau}^{0}\|z(\sigma)\|_{H^{3}}^{2} d \sigma\right)}\left(\|v\|^{2}+\|\nabla v\|^{2}\right) d \tau \\
& +e^{\lambda} \frac{c}{k} \int_{-\infty}^{0} e^{-\tau\left(-\lambda-\frac{2 b^{2}}{\tau} \int_{\tau}^{0}\|z(\sigma)\|_{H^{3}}^{2} d \sigma\right)}\left(\|z\|_{H^{2}}^{2}\|v\|^{2}\right) d \tau \\
& +e^{\lambda} \int_{-\infty}^{0} e^{-\tau\left(-\lambda-\frac{2 b^{2}}{\tau} \int_{\tau}^{0}\|z(\sigma)\|_{H^{3}}^{2} d \sigma\right)} E(\tau) d \tau
\end{aligned}
$$

As in the former discussion, we know that there exists $s_{2}(\omega)<s_{1}(\omega)$ such that for all $s<s_{2}(\omega)$ we have

$$
e^{-s\left(-\lambda-\frac{2 b^{2}}{s} \int_{s}^{0} \sum_{k=1}^{m}\left|z_{k}(\sigma)\right|^{2} d \sigma\right)} \leq e^{\frac{\lambda s}{2}},
$$

which decays exponentially as $s \rightarrow-\infty$. Then there exists $s^{*}<s_{2}(\omega)$ such that for all $s \leq s^{*}$ we have

$$
e^{\lambda} e^{-s\left(-\lambda-\frac{2 b^{2}}{s} \int_{s}^{0}\|z(\sigma)\|_{H^{3}}^{2} d \sigma\right)}\|v(s)\|^{2} \leq \frac{\varepsilon}{4} .
$$

For the second term of the right of the (4.21), there exists $R_{1}>0$ such that for all $k>R_{1}$ we have

$$
e^{\lambda} \frac{c}{k} \int_{-\infty}^{0} e^{-\tau\left(-\lambda-\frac{2 b^{2}}{\tau} \int_{\tau}^{0}\left(\|z(\sigma)\|_{H^{3}}^{2} d \sigma\right)\right.}\left(\|v\|^{2}+\|\nabla v\|^{2}\right) d \tau \leq \frac{\varepsilon}{4}
$$


according to Lemma 4.2.

For the third term of the right of the (4.21), we have

$$
\begin{aligned}
& e^{\lambda} \frac{c}{k} \int_{-\infty}^{0} e^{-\tau\left(-\lambda-\frac{2 b^{2}}{\tau} \int_{\tau}^{0}\left(\|z(\sigma)\|_{H^{3}}^{2}\right) d \sigma\right.}\left(\|z\|_{H^{2}}^{2}\|v\|^{2}\right) d \tau \\
\leq & e^{\lambda} \frac{c}{k} \int_{-\infty}^{0} e^{\frac{\lambda \tau}{4}}\|z\|_{H^{2}}^{2} e^{-\frac{\tau}{2}\left(-\lambda-\frac{2 b^{2}}{\tau} \int_{\tau}^{0}\left(\|z(\sigma)\|_{H^{3}}^{2}\right) d \sigma\right.}\|v\|^{2} d \tau .
\end{aligned}
$$

Since $\sum_{k=1}^{m}\left(\left|z_{k}\right|^{2}+\left|z_{k}\right|^{4}\right)$ grows at most polynomially, we know that $e^{\frac{\lambda \tau}{4}}\|z\|_{H^{2}}^{2}$ is bounded for all $\tau<0$. Therefore, by Lemma 4.1 there exists $R_{2}>0$ such that for all $k>R_{2}$ we have

$$
\begin{aligned}
& e^{\lambda} \frac{c}{k} \int_{-\infty}^{0} e^{-\tau\left(-\lambda-\frac{2 b^{2}}{\tau} \int_{\tau}^{0}\left(\|z(\sigma)\|_{H^{3}}^{2}\right) d \sigma\right.}\left(\|z\|_{H^{2}}^{2}\|v\|^{2}\right) d \tau \\
\leq & e^{\lambda} \frac{c}{k} \int_{-\infty}^{0} e^{-\frac{\tau}{2}\left(-\lambda-\frac{2 b^{2}}{\tau} \int_{\tau}^{0}\left(\|z(\sigma)\|_{H^{3}}^{2}\right) d \sigma\right.}\|v\|^{2} d \tau \leq \frac{\varepsilon}{4} .
\end{aligned}
$$

For the fourth term of the right hand side of (4.21) there exists $R_{3}>0$ such that for all $k>R_{3}$ we have

$$
e^{\lambda} \int_{-\infty}^{0} e^{-\tau\left(-\lambda-\frac{c}{\tau} \int_{\tau}^{0}\left(\|z(\sigma)\|_{H^{3}}^{2}\right) d \sigma\right.} E(\tau) \leq \frac{\varepsilon}{4}
$$

when $k$ is large enough, because $E(t) \leq \varepsilon \sum_{k=1}^{m}\left|z_{k}\right|^{2}$ when $|x|>R_{3}$ and $\sum_{k=1}^{m}\left|z_{k}\right|^{2}$ grows at most polynomially. We then know that for any $\varepsilon>0$, there exists $s^{*}$ and $R^{*}>\sqrt{2} \max \left\{R_{1}, R_{2}, R_{3}\right\}$, such that for all $s \leq s^{*}$ we have

$$
\int_{|x| \geq R^{*}}|v(t)|^{2} d x \leq \int_{|x| \geq \sqrt{2} k}|v(t)|^{2} d x \leq \int_{\mathbb{R}^{2}} \rho|v(t)|^{2} d x \leq \varepsilon, \quad t \in[-1,0] .
$$

The proof is complete.

Lemma 4.5. Let $\phi_{i}(x) \in H^{3}\left(\mathbb{R}^{2}\right), \eta>0$ be given and $u_{s} \in H$ satisfy $\left\|u_{s}\right\| \leq \eta$. Then for every $\varepsilon>0$ and $P$-a.e. $\omega \in \Omega$, there exist $s^{* *}(\omega, \varepsilon) \leq-1$ and $R^{* *}(\omega, \varepsilon)>0$, such that for all $s \leq s^{* *}(\omega, \varepsilon)$ and $R>R^{* *}(\omega, \varepsilon)$, the solution $u(t)$ satisfies

$$
\int_{|x| \geq R^{* *}}|u(t)|^{2} d x \leq \frac{\varepsilon}{4}, \quad t \in[-1,0] .
$$

Proof. Let $s^{*}$ and $R^{*}$ be the constants in Lemma 4.4. Choosing $R^{* *}>R^{*}$ and $s^{* *}<s^{*}$, for all $s \leq s^{* *}$ and $|x| \geq R^{* *}>\sqrt{2} k>R^{*}$ we have

$$
\begin{gathered}
\int_{|x| \geq R^{* *}}|z(t)|^{2} d x=\int_{|x| \geq R^{* *}}\left|\sum_{i=1}^{m} \phi_{i}(x) z_{i}(t)\right|^{2} d x \\
\leq m^{2} \int_{|x| \geq R^{* *}} \sum_{i=1}^{m}\left|\phi_{i}(x)\right|^{2}\left|z_{i}(t)\right|^{2} d x \leq \frac{\varepsilon}{4}, \quad t \in[-1,0],
\end{gathered}
$$

and

$$
\int_{|x| \geq R^{* *}}|v(t)|^{2} d x \leq \frac{\varepsilon}{4}, \quad t \in[-1,0]
$$


Then by (4.23) and (4.24), for all $s \leq s^{* *}$ and $|x| \geq R^{* *}$ we have

$$
\begin{aligned}
\int_{|x| \geq R^{* *}}|u(t)|^{2} d x & =\int_{|x| \geq R^{* *}}|v(t)+z(t)|^{2} d x \\
& \leq 2 \int_{|x| \geq R^{* *}}|v(t)|^{2} d x+2 \int_{|x| \geq R^{*}}|z(t)|^{2} d x \\
& \leq \varepsilon, \quad t \in[-1,0],
\end{aligned}
$$

which completes the proof.

Similar to the arguments in the proofs of Lemma 4.3 and Lemma 4.5, we can obtain the following lemmas. In contrast to Lemma 4.5, the higher regularity for $\phi_{i}$ is necessary in Lemma 4.7 due to the estimates in the higher space $H^{1}\left(\mathbb{R}^{2}\right)$.

Lemma 4.6. Let $\phi_{i}(x) \in H^{3}\left(\mathbb{R}^{2}\right)$ and $v(t)$ be the solution of (3.8)-(3.9). For any given $\eta>0$ and $u_{s} \in H^{1}\left(\mathbb{R}^{2}\right)$ satisfying $\left\|u_{s}\right\| \leq \eta$, there exist random radii $r_{9}(\omega), r_{10}(\omega), r_{11}(\omega)$ and $s_{2}(\omega) \leq-1$, for all $s \leq s_{2}(\omega)$, such that the following inequalities hold P-a.e.:

$$
\begin{aligned}
& \|\Delta v(t)\|^{2} \leq r_{9}(\omega),\|\Delta u(t)\|^{2} \leq r_{10}(\omega), \quad t \in[-1,0], \\
& \int_{-1}^{0}\|\nabla \Delta v(t)\|^{2} d t \leq r_{11}(\omega) .
\end{aligned}
$$

Lemma 4.7. Let $\phi_{i}(x) \in H^{5}\left(\mathbb{R}^{2}\right), \eta>0$ be given and $u_{s} \in H^{1}\left(\mathbb{R}^{2}\right)$ satisfy $\left\|u_{s}\right\| \leq \eta$. Then for every $\varepsilon>0$ and $P$-a.e. $\omega \in \Omega$, there exist $s^{* * *}(\omega, \varepsilon) \leq-1$ and $R^{* * *}(\omega, \varepsilon)>0$, such that for all $s \leq s^{* * *}(\omega, \varepsilon)$ and $R>R^{* * *}(\omega, \varepsilon)$, the solution $u(t)$ satisfies

$$
\int_{|x| \geq R^{* * *}}|\nabla u(t)|^{2} d x \leq \frac{\varepsilon}{4}, \quad t \in[-1,0] .
$$

\section{Random attractors}

In this section, we prove the existence of random attractors for the stochastic dynamical system $S(t, s ; \omega)$ associated with the stochastic hydrodynamical equations in the entire space $\mathbb{R}^{2}$. We first prove the asymptotic compactness of $S$ in $L^{2}\left(\mathbb{R}^{2}\right)$ by using the uniform estimates on the tails of solutions.

Lemma 5.1. Assume that $\phi_{i}(x) \in H^{3}\left(\mathbb{R}^{2}\right)$. Then the random dynamical system $S(t, s ; \omega)$ is asymptotically compact in $L^{2}\left(\mathbb{R}^{2}\right)$; that is, for P-a.e. $\omega \in \Omega$, the sequence $u\left(0, s_{n} ; \omega\right)$ has a convergent subsequence in $L^{2}\left(\mathbb{R}^{2}\right)$ provided $s_{n} \rightarrow-\infty$.

Proof. Let $s_{n} \rightarrow-\infty$. Then by Lemma 4.1, for $P$-a.e. $\omega \in \Omega$, we have that

$$
\left\{u\left(0, s_{n} ; \omega\right)\right\}_{n=1}^{\infty} \text { is bounded in } L^{2}\left(\mathbb{R}^{2}\right) .
$$

Then there is $\xi \in L^{2}\left(\mathbb{R}^{2}\right)$ such that

$$
u\left(0, s_{n} ; \omega\right) \rightarrow \xi \text { weakly in } L^{2}\left(\mathbb{R}^{2}\right) \text { as } s_{n} \rightarrow-\infty .
$$

Next we prove that the weak convergence of (5.1) is actually strong convergence. Given $\varepsilon>0$, by Lemma 4.4 there is $T_{1}(\eta, \omega, \varepsilon)$ and $R(\omega, \varepsilon)$ such that for all $s<T_{1}$ we have

$$
\int_{|x| \geq R}|u(0, s ; \omega)|^{2} d x \leq \frac{\varepsilon}{6}
$$


Since $s_{n} \rightarrow-\infty$, there is $N_{1}(\eta, \omega, \varepsilon)$ such that $s_{n}<N_{1}$ for every $n>N_{1}$. Therefore it follows from (5.2) that for all $n>N_{1}$ we have

$$
\int_{|x| \geq R}\left|u\left(0, s_{n} ; \omega\right)\right|^{2} d x \leq \frac{\varepsilon}{6} .
$$

On the other hand, by Lemma 4.1 and Lemma 4.2 there are $T_{2}(\eta, \omega)$ and $r(\omega)$ such that for all $s<T_{2}$, we have

$$
\left\|u\left(0, s_{n} ; \omega\right)\right\|_{H^{1}\left(\mathbb{R}^{2}\right)}^{2} \leq r(\omega) .
$$

Denote the set $\left\{x \in \mathbb{R}^{2}:|x| \leq R\right\}$ by $Q_{R}$. By the compactness of the embedding $H^{1}\left(Q_{R}\right) \hookrightarrow L^{2}\left(Q_{R}\right)$, it follows from (5.4) that there is a subsequence

$$
u\left(0, s_{n} ; \omega\right) \rightarrow \xi \text { strongly in } L^{2}\left(Q_{R}\right) \text { as } s_{n} \rightarrow-\infty,
$$

which shows that for given $\varepsilon>0$ there exists $N_{3}(\eta, \omega, \varepsilon)$ such that for all $n>N_{3}$,

$$
\left\|u\left(0, s_{n} ; \omega\right)-\xi\right\|_{L^{2}\left(Q_{R}\right)}^{2} \leq \frac{\varepsilon}{3} .
$$

Note that $\xi \in L^{2}\left(\mathbb{R}^{2}\right)$, so there exists $R^{\prime}(\varepsilon)>R$ such that

$$
\int_{|x| \geq R^{\prime}}|\xi|^{2} \leq \frac{\varepsilon}{6}
$$

and

$$
\left\|u\left(0, s_{n} ; \omega\right)-\xi\right\|_{L^{2}\left(Q_{R^{\prime}}\right)}^{2} \leq \frac{\varepsilon}{3} .
$$

Let $N_{4}=\max \left\{N_{1}, N_{3}\right\}$. By (5.3), (5.6), and (5.7), we find that for all $n \geq N_{4}$

$$
\begin{aligned}
\left\|u\left(0, s_{n} ; \omega\right)-\xi\right\|_{L^{2}\left(\mathbb{R}^{2}\right)}^{2} & \leq \int_{|x| \geq R^{\prime}}\left|u\left(0, s_{n} ; \omega\right)-\xi\right|^{2} d x+\int_{|x| \leq R^{\prime}}\left|u\left(0, s_{n} ; \omega\right)-\xi\right|^{2} d x \\
& \leq \varepsilon
\end{aligned}
$$

which shows that

$$
u\left(0, s_{n} ; \omega\right) \rightarrow \xi \text { strongly in } L^{2}\left(\mathbb{R}^{2}\right) \text { as } s_{n} \rightarrow-\infty,
$$

as desired. The proof is complete.

Through a similar discussion, we can prove the asymptotic compactness of $S$ in $H^{1}\left(\mathbb{R}^{2}\right)$ by using the uniform estimates on the tails of solutions.

Lemma 5.2. Assume that $\phi_{i}(x) \in H^{5}\left(\mathbb{R}^{2}\right)$. Then the random dynamical system $S(t, s ; \omega)$ is asymptotically compact in $H^{1}\left(\mathbb{R}^{2}\right)$; that is, for $P$-a.e. $\omega \in \Omega$, the sequence $u\left(0, s_{n} ; \omega\right)$ has a convergent subsequence in $H^{1}\left(\mathbb{R}^{2}\right)$ provided $s_{n} \rightarrow-\infty$.

TheOREM 5.1. Assume that $\phi_{i}(x) \in H^{5}\left(\mathbb{R}^{2}\right)$. Then the stochastic dynamical system $S(t, s ; \omega)$ has a unique random attractor in $H^{1}\left(\mathbb{R}^{2}\right)$.

Proof. Notice that $S(t, s ; \omega)$ has a random absorbing set $A(\omega)$ in $H^{1}\left(\mathbb{R}^{2}\right)$ by Lemma 4.1 and Lemma 4.3 , and is asymptotically compact in $L^{2}\left(\mathbb{R}^{2}\right)$ and $H^{1}\left(\mathbb{R}^{2}\right)$ by Lemma 5.1 and Lemma 5.2. Hence the existence of a unique random attractor for 
$S$ follows from Theorem 2.6 immediately; see also [18]. The proof of Theorem 5.1 is complete.

In fact, the regularity of the random attractor is also obtained in the course of the proof. From Lemma 4.6 and Lemma 4.7, we can see that the attractor can be much smoother than the initial data, which implies the asymptotic smoothing effect of the equation in a probabilistic sense.

Note that the above investigation mainly worked with the entire plane. If we consider the unbounded domain, it is trivial that the results above can be extended under the imposed condition $u=0$ for an infinite strip domain, $x \in \partial \Omega$. For more details of the infinite strip, one can refer to [2], where the authors investigated the existence of a compact attractor in an unbounded channel. In the cases of others, we will further do some discussion for the extra difficulties produced by boundary terms in the future.

Acknowledgement. This paper is supported by NSFC Grant No.11061003; Guangxi NSF Grant No.0832065; Guangxi Education Institution Scientific Research Item No.200911LX150; The Fundamental Research Funds for the Central Universities N0.2010QS04.

\section{REFERENCES}

[1] P. W. Bates, K. Lu, and B. Wang, Random attractors for stochastic reaction-diffusion equations on unbounded domains, J. Diff. Eqs., 246, 845-869, 2009.

[2] F. Bloom and W. Hao, Regularization of a non-Newtonian system in an unbounded channel: Existence of a maximal compact attractor, Nonlinear Anal. TMA, 43, 743-766, 2001.

[3] A. de Bouard and A. Debussche, On the stochatic Korteweg-de Vires equation, J. Funct. Anal., $154,215-251,1998$.

[4] A. de Bouard and A. Debussche, The stochastic nonlinear Schrödinger equation in $H^{1}$, Stoch. Anal. Appl., 21(1), 97-126, 2003.

[5] H. Crauel, A. Debussche, and F. Flandoli, Random attractors, J. Dynam. Diff. Eqs., 9, 307-341, 1997.

[6] H. Crauel and F. Flandoli, Attractors for random dynamical systems, Probab. Theory Related Fields, 100, 365-393, 1994.

[7] B. Guo and Y. Han, Global smooth solution of hydrodynamical equations for the Heisenberg paramagnet, Math. Meth. Appl. Sci., 27, 181-191, 2004.

[8] B. Guo, X. Pu, and Y. Han, On the stochastic hydrodynamical equation in Hersenberg paramagnet, to appear.

[9] B. Guo, C. Guo, and X. Pu, Random attractors for a stochastic hydrodynamical equation in Hersenberg paramagnet, Acta Mathematica Scientia, 31B, 529-540, 2011.

[10] B. Guo and S. Ding, Landau-Lifshitz Equations, Frontiers of Research with the Chinese Academy of Sciences, Singapore: World Scientific, 1, 2008.

[11] J.U. Kim, On the stochastic porous medium equation, J. Diff. Eqs., 220, 163-194, 2006.

[12] L.D. Landau and E.M. Lifshitz, On the theory of the dispersion of magnetic permeability in ferromagnetic bodies, Phys. Zeitsch. der Sow., 8, 153-169, 1935.

[13] G. Da Prato, A. Debussche, and R. Temam, Stochastic Burgers' equation, NoDEA., 1, 389-402, 1994.

[14] G. Da Prato and J. Zabczyk, Ergodicity for Infinite Dimensional System, Cambridge University Press, 1996.

[15] J.C. Robinson, Infinite Dimensional Dynamical Systems, Cambridge Univ. Press, Cambridge, UK, 2001.

[16] R. Temam, Infinite-Dimensional Dynamical Systems in Mechanics and Physics, SpingerVerlag, New York, 1997.

[17] B. Wang, Attractors for reaction-diffusion equations in unbounded domains, Phys. D, 128, 41-52, 1999.

[18] B. Wang and X. Gao, Random attractors for wave equations on unbounded domains, Disc. Cont. Dyn. Sys., Supplement, 800-809, 2009. 